\title{
Performance of rupture-related morphological parameters in posterior communicating artery aneurysms with fetal-type variant
}

\author{
S. Chen ${ }^{1 *}$, C. Li ${ }^{3 *}$, C. Karmonik ${ }^{4}$, Y. Cheng ${ }^{1}$, N. Lv \\ 'Shanghai Interventional Medical Device Engineering Technology Research Centre, \\ University of Shanghai for Science and Technology, Shanghai, China \\ 2Department of Neurosurgery, Changhai Hospital, Second Military Medical University, Shanghai, China \\ ${ }^{3}$ Department of Geriatrics, Dongying New District Hospital, Dongying, China \\ ${ }^{4} \mathrm{MRI}$ Core, Houston Methodist Research Institute, Houston, Texas, United States
}

[Received: 12 October 2021; Accepted: 5 November 2021; Early publication date: 9 November 2021]

Background: The aim of the study was to investigate the impact of fetal-type posterior cerebral artery ( $F P C A$ ) variant on morphological parameters of posterior communicating artery (PComA) aneurysms for rupture risk assessment.

Materials and methods: A total of 98 PComA aneurysms (62 ruptured and 36 unruptured) in 98 consecutive patients were reviewed. Morphological parameters were calculated including aneurysm size, aspect ratio (AR), size ratio (SR), dome-to-neck ratio, bottleneck factor and inflow angle. Performances of morphological parameters to discriminate rupture status were compared between aneurysms with or without $P P C A$.

Results: Fetal-type posterior cerebral artery variant was determined in 39 (39.8\%, 25 ruptured and 14 unruptured) lesions. The ruptured group revealed a significantly larger size $(p=0.004), A R(p=0.003), S R(p=0.001)$, and inflow angle $(p<0.001)$. For the aneurysms without fPCA, all morphological parameters were significantly different between ruptured and unruptured aneurysms $(p<0.05)$; for the aneurysms with IPCA, only inflow angle ( $p=0.001)$ was significantly related with the rupture status. Multivariate analysis showed that $S R$ ( $p=0.035$ and $p=0.011)$ and inflow angle $(p=0.001$ and $p=0.028)$ were independent rupture risk factors for the total cohort and the aneurysms without $f P C A$; while only inflow angle $(p=0.004)$ revealed to be independently related with rupture status of aneurysms without fPCA.

Conclusions: The performances of morphological parameters to discriminate rupture status were different between PComA aneurysms with and without PPCA variants. Inflow angle might be a reliable predictor for rupture risk of PComA aneurysms. (Folia Morphol 2023; 82, 1: 30-36)

Key words: intracranial aneurysm, rupture, morphology

Address for correspondence: Dr. N. Lv, Department of Neurosurgery, Changhai Hospital, 168 Changhai Road, Shanghai, China, 200433, tel:+86 21-31161784, fax: +86 21-31161784, e-mail: Ivnan2008@163.com

${ }^{*} \mathrm{~S}$. Chen and C. Li contributed equally to this work and should be considered as co-first authors.

This article is available in open access under Creative Common Attribution-Non-Commercial-No Derivatives 4.0 International (CC BY-NC-ND 4.0) license, allowing to download articles and share them with others as long as they credit the authors and the publisher, but without permission to change them in any way or use them commercially. 


\section{INTRODUCTION}

Advances in neuroimaging techniques are leading to an increasing number of incidentally detected unruptured intracranial aneurysms (IAs) [1, 2]. Despite the adverse consequences of morbidity and mortality of subarachnoid haemorrhage $(\mathrm{SAH})$ caused by a ruptured IA, the annual incidence rate of such an event is comparable to or even lower than the risk of preventive treatment [18]. Therefore, it is important to identify risk factors for rupture so that treatment strategies for the individualised patient may be optimised.

Accounting for $15-25 \%$ of all IAs, posterior communicating artery (PComA) aneurysms are one of the most frequent occurring types and have larger rupture risk than aneurysms of other segments of the internal carotid artery (ICA) [3]. Several location-specific studies on PComA aneurysms suggest that the morphology of the aneurysm and its involved arteries may serve as a potential discriminant for rupture status $[8,10]$. Several anatomic vascular variants of the ICA-PComA bifurcation exist at this location [16]. Morphological parameters calculated based on the size or direction of aneurysmal sac and its parent arteries only may not consider the influence of these vascular variants. This might partially explain why even location-specific studies considering vessel wall characteristics and peri-aneurysmal environment did not achieve consensus conclusions $[8,10]$.

Fetal-type posterior cerebral artery (fPCA) is one of the most frequent types of vascular variants of the ICA-PComA bifurcation, occurring in $4-29 \%$ of the population $[5,16]$. To maintain adequate blood flow in the posterior circulation, PComAs with PPCA variants tend to have a larger diameter and different angulations, which might cause distinctive morphological features of aneurysms originated from this kind of ICA-PComA bifurcation. In this study, we reviewed the morphological parameters of PComA aneurysms with and without PPCA, to investigate the impact of this variant on rupture risk assessment of PComA aneurysms.

\section{MATERIALS AND METHODS}

This retrospective study was approved by our institutional review board, and the requirement for informed consent was waived. The patients' information was anonymised and de-identified before analysis.

\section{Patients}

We retrospectively reviewed 163 consecutive patients with diagnosed PComA aneurysms between January 2015 and December 2016 at our institution. The PComA aneurysms were determined and measured using three-dimensional rotational angiography (3DRA). Only aneurysms that involved both ICA and PComA were retained in the study to achieve uniform definitions of morphological parameters. SAH patients with multiple aneurysms were excluded to avoid misjudgement of aneurysmal rupture status. Finally, 98 PComA aneurysms in 98 patients were retained, including 62 ruptured and 36 unruptured lesions. Of the 36 patients in the unruptured group, 14 exhibited mild headache or dizziness, 7 suffered from ischaemic events, 3 exhibited symptoms of oculomotor nerve palsy, and the other 12 aneurysms were detected incidentally without symptoms of cerebrovascular disease.

The clinical characteristics were collected as the following: age, sex, hypertension, diabetes mellitus, and current smoking status. Hypertension was defined as taking antihypertensive agents, a systolic blood pressure $\geq 140 \mathrm{mmHg}$, or a diastolic blood pressure $\geq 90 \mathrm{mmHg}$. Diabetes mellitus was defined as taking antidiabetic agents, treatment with insulin injections, a fasting plasma glucose level $\geq 126 \mathrm{mg} / \mathrm{dL}$, a random plasma glucose level of $>200 \mathrm{mg} / \mathrm{dL}$, or a haemoglobin $\mathrm{A} 1 \mathrm{c}$ level $\geq 6.5 \%$. Current smoker was defined as those who had smoked at least 100 cigarettes during their lifetime and reported smoking every day or some days before being admitted.

\section{Radiological findings and morphological calculations}

The 3DRA was performed using the Artis zee Biplane angiographic system (VC14; Siemens, Erlangen, Germany). The acquired 3DRA data were transferred to the syngo $X$ Workplace (VB15; Siemens) for reconstruction of the 3D internal carotid artery vessel tree and exported in a stereolithography format to Meshmixer 3.0 software (Autodesk Inc., San Francisco, CA, USA). First, we defined the neck plane as the location where the aneurysmal sac pouched outward from the parent vessel. Afterward, the models were divided into the aneurysm dome and the inlet and outlet planes of the parent artery, and then exported in stereolithography format. These formats were imported into Matlab 7.0 (MathWorks, Natick, Massachusetts, USA), which was used to calculate and visualise the morphological parameters. 


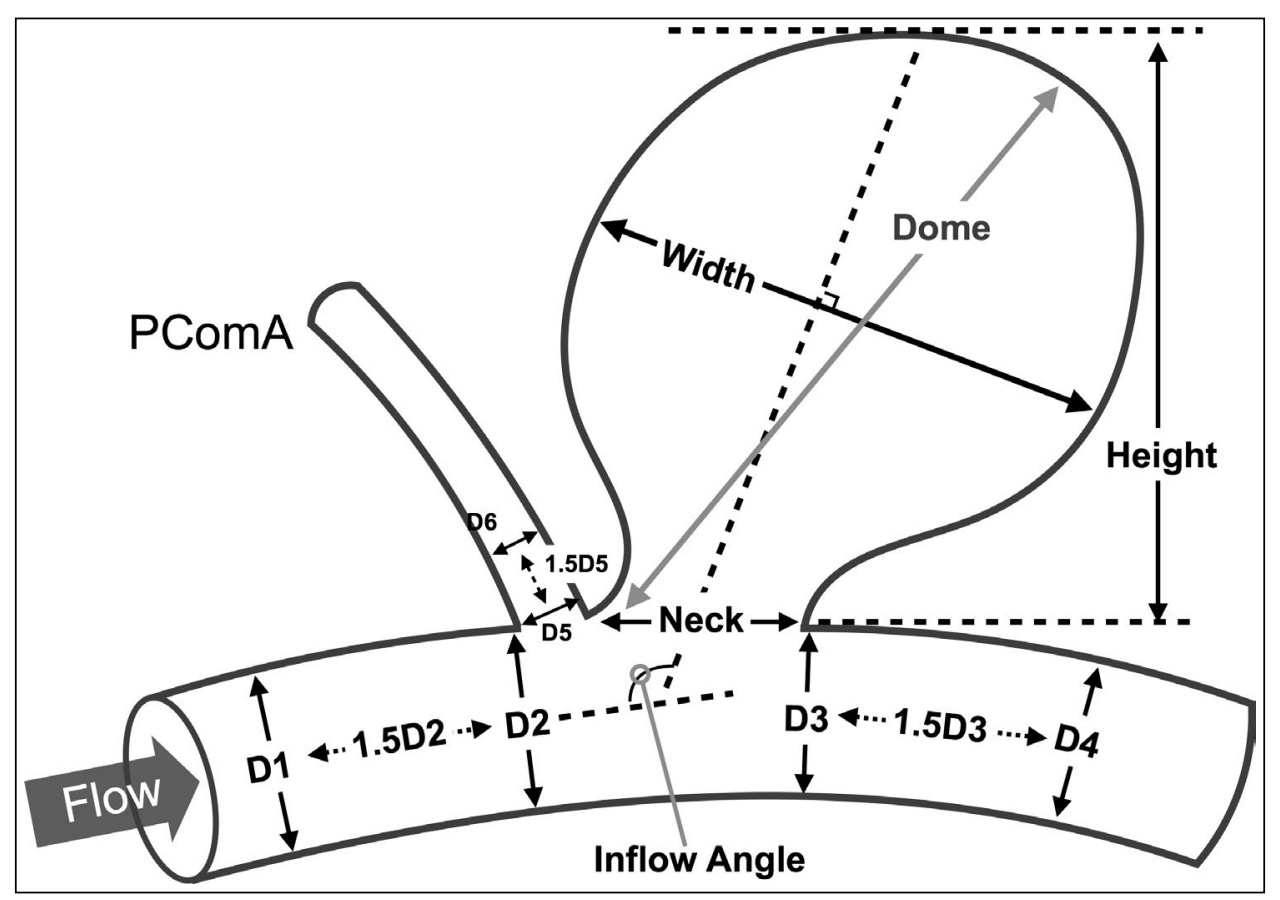

Figure 1. Definitions of morphological parameters; Size = Dome; Aspect Ratio $=$ Height/Neck; Size Ratio $=6 \mathrm{Dome} /(\mathrm{D} 1+\mathrm{D} 2+\mathrm{D} 3+\mathrm{D} 4+\mathrm{D} 5+$ +D6); Diameter of PComA = (D5+D6)/2; Dome-to-Neck Ratio = Dome/Neck; Bottle-Neck Factor = Width/Neck; PComA - posterior communicating artery.

Morphological parameters of the PComA aneurysms were derived from these models as described in previously (Fig. 1) [2, 8]. An fPCA variant was defined when PcomA has the same calibre as the P2 segment of the PCA and is associated with an atrophic P1 segment. The size of the aneurysm dome was defined as the maximum diameter of the aneurysm dome. Dome height was the longest dimension from the neck to the dome tip, and the dome width was measured perpendicular to the dome height. Aspect ratio (AR) was computed by dividing the dome height by neck width. Size ratio (SR) was calculated by dividing size by the average diameter of the parent artery and dome-to-neck ratio (DN) by dividing size by neck width. Bottleneck factor (BNF) was defined as the ratio of dome width to neck width. Inflow angle was the angle between inflow and the aneurysm's main axis from the centre of the neck to the tip of the dome.

\section{Statistical analysis}

Statistical analyses were performed using Microsoft Excel 2003 and SAS 9.1 (SAS Institute, Cary, North Carolina, USA). Variables were expressed as median (interquartile range) or number of patients (\%) as appropriate. Analysis was performed for the total cohort, aneurysms with FPCA, and aneurysms without IPCA to compare the performance of morphological parame- ters in different vascular configurations, respectively. In univariate analysis, a Mann-Whitney $U$ test was used for measurement data, and the chi-square test was performed for cross-tabulation. Multivariate logistic regression (backward elimination) was used to identify the independent risk factors. $\mathrm{P}<0.05$ (two-sided) was the criterion for statistical significance.

\section{RESULTS}

\section{Risk factors for PComA aneurysms rupture}

The clinical and morphological characteristics of the patients and PComA aneurysms are shown in Table 1. The patients' ages ranged from 42 to 82 , with a median age of 61 years. Twenty-three were males and 73 were females. The demographic characteristics and medical history showed no significant differences between the ruptured and unruptured groups (Table 2).

The size of PComA aneurysms ranged from 1.23 to $6.84 \mathrm{~mm}$, with a median size of $4.36 \mathrm{~mm}$. The ruptured PComA aneurysms had a significantly larger size $(p=0.004)$, AR $(p=0.003), S R(p=0.001)$ and inflow angle $(p<0.001)$. Other morphological characteristics, including DN $(p=0.113)$, BNF ( $p=$ $=0.089)$, and diameter of PComA $(p=0.206)$ did not exhibit statistical significance between the ruptured and unruptured groups. 
Table 1. Clinical and morphological characteristics of the patients and aneurysms

\begin{tabular}{lcccc}
\hline & $\begin{array}{c}\text { All } \\
(\mathbf{n = 9 8})\end{array}$ & $\begin{array}{c}\text { Fetal-type PComA } \\
(\mathbf{n}=\mathbf{3 9})\end{array}$ & $\begin{array}{c}\text { Non-fetal-type PComA } \\
(\mathbf{n}=\mathbf{5 9})\end{array}$ & $\mathbf{P}$ \\
\hline Age [years] & $61(52,66)$ & $62(52,69)$ & $60(52,65)$ & 0.311 \\
Male & $23(23.5)$ & $8(20.5)$ & $15(25.4)$ & 0.574 \\
Hypertension & $49(50.0)$ & $23(59.0)$ & $26(44.1)$ & 0.149 \\
Diabetes & $11(11.2)$ & $6(15.4)$ & $5(8.5)$ & 0.463 \\
Current smoking & $7(7.1)$ & $2(5.1)$ & $5(8.5)$ & 0.819 \\
Size [mm] & $4.48(3.56,5.57)$ & $4.45(3.92,5.56)$ & $4.49(3.18,5.60)$ & 0.511 \\
Aspect ratio & $1.02(0.81,1.35)$ & $1.00(0.82,1.36)$ & $1.02(0.80,1.32)$ & 0.635 \\
Size ratio & $1.69(1.29,1.95)$ & $1.61(1.32,1.96)$ & $1.70(1.29,1.94)$ & 0.752 \\
Dome to neck ratio & $1.08(0.92,1.37)$ & $1.07(0.89,1.47)$ & $1.10(0.92,1.37)$ & 0.502 \\
Bottleneck factor & $1.16(0.94,1.37)$ & $1.27(0.97,1.42)$ & $1.11(0.90,1.32)$ & 0.176 \\
Inflow angle & $113.0(97.6,127.9)$ & $114.6(101.5,124.2)$ & $108.4(95.9,127.9)$ & 0.234 \\
PComA diameter [mm] & $1.32(0.04,1.95)$ & $1.97(1.70,2.50)$ & $0.73(0.04,1.35)$ & $<\mathbf{0 . 0 0 1}$ \\
\hline
\end{tabular}

Data was expressed as median $(25 \%, 75 \%)$; PComA — posterior communicating artery

Table 2. Univariate analysis of rupture-related parameters in aneurysms with or without fetal-type variant

\begin{tabular}{|c|c|c|c|c|c|c|c|c|c|}
\hline & \multicolumn{2}{|c|}{ All } & \multirow[t]{2}{*}{$\mathbf{r}$} & \multicolumn{2}{|c|}{ Fetal-type PCA } & \multirow[t]{2}{*}{$\mathbf{P}$} & \multicolumn{2}{|c|}{ Non-fetal-type PCA } & \multirow[t]{2}{*}{$\mathbf{P}$} \\
\hline & $\begin{array}{l}\text { Ruptured } \\
(\mathrm{n}=62)\end{array}$ & $\begin{array}{l}\text { Unruptured } \\
(\mathrm{n}=36)\end{array}$ & & $\begin{array}{l}\text { Ruptured } \\
(\mathrm{n}=25)\end{array}$ & $\begin{array}{l}\text { Unruptured } \\
(\mathrm{n}=14)\end{array}$ & & $\begin{array}{l}\text { Ruptured } \\
(\mathrm{n}=37)\end{array}$ & $\begin{array}{l}\text { Unruptured } \\
(n=22)\end{array}$ & \\
\hline Size $[\mathrm{mm}]$ & $\begin{array}{c}4.54 \\
(3.97,5.81)\end{array}$ & $\begin{array}{c}4.07 \\
(2.74,4.94)\end{array}$ & 0.004 & $\begin{array}{c}4.39 \\
(3.81,5.77)\end{array}$ & $\begin{array}{c}4.52 \\
(3.92,5.58)\end{array}$ & 0.942 & $\begin{array}{c}4.61 \\
(4.16,5.86)\end{array}$ & $\begin{array}{c}3.05 \\
(2.41,4.66)\end{array}$ & 0.001 \\
\hline Aspect ratio & $\begin{array}{c}1.13 \\
(0.89,1.37)\end{array}$ & $\begin{array}{c}0.85 \\
(0.71,1.24)\end{array}$ & 0.003 & $\begin{array}{c}1.00 \\
(0.81,1.36)\end{array}$ & $\begin{array}{c}1.05 \\
(0.83,1.36)\end{array}$ & 0.942 & $\begin{array}{c}1.18 \\
(0.91,1.49)\end{array}$ & $\begin{array}{c}0.77 \\
(0.61,1.02)\end{array}$ & $<0.001$ \\
\hline Size ratio & $\begin{array}{c}1.77 \\
(1.42,2.17)\end{array}$ & $\begin{array}{c}1.46 \\
(0.90,1.80)\end{array}$ & 0.001 & $\begin{array}{c}1.61 \\
(1.28,2.22)\end{array}$ & $\begin{array}{c}1.60 \\
(1.40,1.89)\end{array}$ & 0.942 & $\begin{array}{c}1.80 \\
(1.59,2.13)\end{array}$ & $\begin{array}{c}0.98 \\
(0.79,1.78)\end{array}$ & $<0.001$ \\
\hline Dome to neck ratio & $\begin{array}{c}1.10 \\
(0.94,1.40)\end{array}$ & $\begin{array}{c}1.02 \\
(0.87,1.24)\end{array}$ & 0.113 & $\begin{array}{c}1.07 \\
(0.89,1.47)\end{array}$ & $\begin{array}{c}1.14 \\
(0.84,1.35)\end{array}$ & 0.919 & $\begin{array}{c}1.25 \\
(0.99,1.40)\end{array}$ & $\begin{array}{c}1.01 \\
(0.89,1.23)\end{array}$ & 0.047 \\
\hline Bottleneck factor & $\begin{array}{c}1.22 \\
(1.04,1.40)\end{array}$ & $\begin{array}{c}1.10 \\
(0.90,1.32)\end{array}$ & 0.089 & $\begin{array}{c}1.27 \\
(0.94,1.39)\end{array}$ & $\begin{array}{c}1.25 \\
(1.06,1.55)\end{array}$ & 0.633 & $\begin{array}{c}1.21 \\
(1.04,1.43)\end{array}$ & $\begin{array}{c}0.96 \\
(0.83,1.16)\end{array}$ & 0.003 \\
\hline Inflow angle & $\begin{array}{c}118.5 \\
(105.7,132.2)\end{array}$ & $\begin{array}{c}98.7 \\
(89.9,113.1)\end{array}$ & $<0.001$ & $\begin{array}{c}120.5 \\
(109.9,134.4)\end{array}$ & $\begin{array}{c}101.0 \\
(93.5,113.7)\end{array}$ & 0.001 & $\begin{array}{c}116.0 \\
(103.9,130.9)\end{array}$ & $\begin{array}{c}95.7 \\
(86.6,111.6)\end{array}$ & 0.001 \\
\hline
\end{tabular}

Data was expressed as median $(25 \%, 75 \%)$; PCA — posterior cerebral artery

Table 3. Multivariate analysis of rupture-related parameters in aneurysms with or without fetal-type variant

\begin{tabular}{lcccc}
\hline Group & $\begin{array}{c}\text { Independent } \\
\text { risk factor }\end{array}$ & $\begin{array}{c}\text { Odds } \\
\text { ratio }\end{array}$ & $\mathbf{9 5 \%} \mathbf{C l}$ & $\mathbf{P}$ \\
\hline All $(\mathrm{n}=98)$ & Size ratio & 1.625 & $1.034-2.553$ & 0.035 \\
& Inflow angle & 2.286 & $1.417-3.690$ & 0.001 \\
$\begin{array}{l}\text { Fetal PCA } \\
\text { (n=39) }\end{array}$ & Inflow angle & 3.223 & $1.444-7.197$ & 0.004 \\
$\begin{array}{l}\text { Non-fetal PCA } \\
\text { (n=59) }\end{array}$ & Size ratio & 2.378 & $1.216-4.650$ & 0.011 \\
& Inflow angle & 2.086 & $1.082-4.020$ & 0.028 \\
\hline
\end{tabular}

$\mathrm{Cl}$ — confidential interval; PCA — posterior cerebral artery

Multivariate logistic regression was performed to identify the independent risk factors of PComA aneu- rysm rupture using a backward elimination process. All significant parameters that were significant in the univariate analysis were included. The result showed that SR (odds ratio [OR]: 1.625; 95\% confidence interval $[\mathrm{Cl}]: 1.034-2.553 ; \mathrm{p}=0.04)$ and inflow angle (OR: 2.286; 95\% Cl: 1.417-3.690; $p<0.01$ ) were independently associated with the rupture status of PComA aneurysms (Table 3).

\section{Impact of fPCA on morphological parameters}

Of the total 98 patients, unilateral fPCA with ipsilateral PComA aneurysm was observed in 33 patients and bilateral fPCA in 6 patients. In this group, 25 (64.1\%) aneurysms were ruptured and 14 (35.9\%) 
were unruptured; while in aneurysms without PPCA variant, 37 (62.7\%) were ruptured and 22 (37.3\%) were unruptured. Between the fPCA and non-fPCA groups, the baseline characteristics and morphological parameters showed no significant differences, except that the diameters of PComA were higher in the fPCA group $(p<0.01)$ (Table 1).

Then the parameters were further analysed to investigate their relationship with aneurysm rupture in both FPCA group and non-fPCA group relatively (Tables 2, 3). In the fPCA group, only the inflow angle was significantly different between ruptured and unruptured lesions, and further logistic regression analysis showed it as an independent risk factor for rupture (OR: $3.223 ; 95 \% \mathrm{Cl}: 1.444-7.197 ; \mathrm{p}=0.004)$. While in non-fPCA group, all morphological parameters were significantly associated with the rupture status of PComA aneurysms $(p<0.05)$. Logistic regression analysis showed the independent risk factors for rupture were SR (OR: $2.378 ; 95 \% \mathrm{Cl}: 1.216-4.650$; $\mathrm{p}=0.011$ ) and inflow angle (OR: $2.086 ; 95 \% \mathrm{Cl}$ : 1.082-4.020; $p=0.028$ ).

\section{DISCUSSION}

In this study, the performances of morphological parameters in discriminating rupture status were compared between PComA aneurysms with and without fetal-type variant of PCA. The results indicated that the arterial variant might have an impact on the morphological analysis for establishing IA rupture risk. Among the parameters included in the study, inflow angle was found to be a statistically significant rupture predictor for PComA aneurysms.

Unruptured intracranial aneurysms are increasingly detected, which emphasizes the necessity of reliable methods for estimating IA rupture risk [17, 18]. Various mechanisms, including morphology, haemodynamics, and wall inflammation, were reported to be associated with IA rupture $[4,8,10$, 11]. Among them, the morphology of $I A$ and its parent arteries might be the most feasible for clinical use because of its simple and quantitative nature. Increasing numbers of morphological parameters have been introduced and may be roughly classified into the following groups: 1) size-related ratios within the aneurysm itself, such as $A R$, which is the ratio of aneurysm height to neck; 2 ) size-related ratios of the aneurysm and its parent artery, such as SR; and 3) other spatial relationships between the aneurysms and its parent artery, such as inflow angle. The utility of these morphological parameters might be affected by other factors not included in the morphological analysis, such as the location of IA [7, 18]. Location-specific designs, such as employed here, are therefore preferred in studies on rupture risk prediction of IAs $[8,10]$. Only PComA aneurysms were considered, which are one of the most common types in clinical practice. Although avoiding biases by distinctive vessel wall characteristics and peri-aneurysmal environment of IAs with this approach, the controversial status of IA rupture prediction remains unchanged. Further sensitizing IA rupture risk investigation to the influence of size and configuration of involved arteries, i.e. vascular variants, may serve as a means to address this controversy. The various anatomic configurations of ICA-PComA bifurcations make PComA aneurysms a well-suited model system to study the effect of these vascular variants on the morphological analysis and thus IA rupture risk. FPCAs is one of the most common variants of the circle of Willis, which has been detected in $4-29 \%$ of the general population $[5,16]$. Although some studies revealed aneurysms occurred more frequently in patients with this type of variant, few evidences have been obtained on its effect on PComA aneurysm rupture prediction [14]. Therefore, we specified our analysis on PComA aneurysms with fPCA to investigate the impact of arterial variants on the performance of morphological parameters. Interestingly, our results show that the size-related parameters (size, AR, SR, DN, BNF) that were significant between ruptured and unruptured aneurysms in non-fPCA group, lost their discriminating power in the PPCA group; and only the inflow angle was significantly related with rupture status of aneurysms of the FPCA variant.

According to the International Study of Unruptured Intracranial Aneurysms (ISUIA), aneurysmal size was closely associated with IA rupture and the rupture rate of IAs smaller than $7 \mathrm{~mm}$ was very low [18]. However, we observed in this study that the median size of ruptured aneurysms was even lower than $5 \mathrm{~mm}$. This indicates that size alone might not be adequate for accurate rupture prediction. In addition, other ratio parameters focusing on the aneurysm itself (AR, $D N$, and BNF) were shown not to be independently associated with IA rupture. Only SR was retained as an independent rupture risk factor in the total cohort and in the non-fPCA group, highlighting the importance of the relationship between aneurysmal sac and its parent artery in aneurysm rupture risk assessment. 
Initially introduced by Dhar et al. [2], SR has been one of the most studied parameters and suggested as a reliable discriminant of rupture status. Kashiwazaki et al. [6] demonstrated that SR could highly predict the rupture risk of IAs, especially for small ones $(<5 \mathrm{~mm})$. Rahman et al. [12] also confirmed prospectively the correlation between SR and aneurysm rupture status. According to a haemodynamic study of Tremmel et al. [15] more complex flow, multiple vortices, and lower wall shear stress were observed in aneurysm models with larger SR. SR not being an independent risk factor for IA rupture in the PPCA group may explain the existence of larger PComAs in the PPCA group.

Inflow angle was the only parameter independently associated with aneurysmal rupture status in both FPCA and non-fPCA. This emphasis on the spatial relationship between the aneurysmal sac and its parent arteries in respect to rupture is consistent with several previous studies [1, 8]. Baharoglu et al. [1] related increasing inflow angle to higher inflow velocity and greater wall shear stress magnitude and spatial gradients in the inflow zone and dome, as well as a greater transmission of kinetic energy into the distal portion of the dome. These haemodynamic features may be important factors that increase the risk of rupture. Inflow angle was also revealed to be associated with irregular haemodynamic patterns [9]. The role of the inflow angle for clinical IA rupture prediction should be investigated in future prospective studies with larger sample size.

\section{Limitations of the study}

The present study does have several limitations. Firstly, as a retrospect and single-institutional study, there is an inherent bias selection of PComA aneurysms. Secondly, the relatively small and unbalanced sample size of both groups might affect the analysis. Due to this limited sample size, only the most widely studied morphological parameters were studied to ensure the reliability of the multivariate analysis. In addition, we assumed that the morphology of the PComA aneurysm did not change significantly after rupture, which could affect the accuracy of the results [13].

\section{CONCLUSIONS}

Performance of morphological parameters in discriminating rupture status is different between PComA aneurysms with and without PPCA variants.
Inflow angle might be a reliable discriminant for the rupture status of PComA aneurysms.

\section{Funding}

This study was funded by the National Research and Development Project of Key Chronic Diseases (Grant No. 2016YFC1300700) and National Natural Science Foundation of China (Grant Nos. 81701775 and 81771264).

\section{Conflict of interest: None declared}

\section{REFERENCES}

1. Baharoglu MI, Schirmer CM, Hoit DA, et al. Aneurysm inflow-angle as a discriminant for rupture in sidewall cerebral aneurysms: morphometric and computational fluid dynamic analysis. Stroke. 2010; 41(7): 1423-1430, doi: 10.1161/STROKEAHA.109.570770, indexed in Pubmed: 20508183.

2. Dhar S, Tremmel M, Mocco J, et al. Morphology parameters for intracranial aneurysm rupture risk assessment. Neurosurgery. 2008; 63(2): 185-197, doi: 10.1227/01. neu.0000316847.64140.81.

3. Forget TR, Benitez R, Veznedaroglu E, et al. A review of size and location of ruptured intracranial aneurysms. Neurosurgery. 2001; 49(6): 1322-1325, doi: 10.1097/00006123200112000-00006, indexed in Pubmed: 11846931.

4. Frösen J, Piippo A, Paetau A, et al. Remodeling of saccular cerebral artery aneurysm wall is associated with rupture: histological analysis of 24 unruptured and 42 ruptured cases. Stroke. 2004; 35(10): 2287-2293, doi: 10.1161/01.STR.0000140636.30204.da, indexed in Pubmed: 15322297

5. Horikoshi T, Akiyama I, Yamagata Z, et al. Magnetic resonance angiographic evidence of sex-linked variations in the circle of willis and the occurrence of cerebral aneurysms. J Neurosurg. 2002; 96(4): 697-703, doi: 10.3171/ jns.2002.96.4.0697, indexed in Pubmed: 11990810.

6. Kashiwazaki $D$, Kuroda S. Size ratio can highly predict rupture risk in intracranial small $(<5 \mathrm{~mm})$ aneurysms. Stroke. 2013; 44(8): 2169-2173, doi: 10.1161/STROKEAHA.113.001138, indexed in Pubmed: 23743979.

7. Korja $\mathrm{M}$, Lehto $\mathrm{H}$, Juvela $\mathrm{S}$, et al. Lifelong rupture risk of intracranial aneurysms depends on risk factors: a prospective Finnish cohort study. Stroke. 2014; 45(7): 1958-1963, doi: 10.1161/STROKEAHA.114.005318, indexed in Pubmed: 24851875

8. Lv N, Feng Z, Wang C, et al. Morphological risk factors for rupture of small $(<7 \mathrm{~mm})$ posterior communicating artery aneurysms. World Neurosurg. 2016; 87: 311-315, doi: 10.1016/j.wneu.2015.12.055, indexed in Pubmed: 26724608.

9. Lv N, Wang C, Karmonik C, et al. Morphological and hemodynamic discriminators for rupture status in posterior communicating artery aneurysms. PLoS One. 2016; 11(2): e0149906, doi: 10.1371/journal.pone.0149906, indexed in Pubmed: 26910518. 
10. Matsukawa H, Fujii M, Akaike G, et al. Morphological and clinical risk factors for posterior communicating artery aneurysm rupture. J Neurosurg. 2014; 120(1): 104-110, doi: 10.3171/2013.9.JNS13921, indexed in Pubmed: 24160476.

11. Miura $Y$, Ishida $F$, Umeda $Y$, et al. Low wall shear stress is independently associated with the rupture status of middle cerebral artery aneurysms. Stroke. 2013; 44(2): 519-521, doi: 10.1161/STROKEAHA.112.675306, indexed in Pubmed: 23223503.

12. Rahman M, Smietana J, Hauck E, et al. Size ratio correlates with intracranial aneurysm rupture status: a prospective study. Stroke. 2010; 41(5): 916-920, doi: 10.1161/ STROKEAHA.109.574244, indexed in Pubmed: 20378866.

13. Schneiders JJ, Marquering HA, van den Berg R, et al. Rupture-associated changes of cerebral aneurysm geometry: high-resolution 3D imaging before and after rupture. AJNR Am J Neuroradiol. 2014; 35(7): 1358-1362, doi: 10.3174/ ajnr.A3866, indexed in Pubmed: 24557706.

14. Songsaeng D, Geibprasert S, Willinsky R, et al. Impact of anatomical variations of the circle of Willis on the incidence of aneurysms and their recurrence rate follow- ing endovascular treatment. Clin Radiol. 2010; 65(11): 895-901, doi: 10.1016/j.crad.2010.06.010, indexed in Pubmed: 20933644.

15. Tremmel M, Dhar S, Levy $E$, et al. Influence of intracranial aneurysm-to-parent vessel size ratio on hemodynamics and implication for rupture. Neurosurgery. 2009; 64(4): 622-631, doi: 10.1227/01.neu.0000341529.11231.69.

16. van Raamt AF, Mali WP, van Laar PJ, et al. The fetal variant of the circle of Willis and its influence on the cerebral collateral circulation. Cerebrovasc Dis. 2006; 22(4): 217-224, doi: 10.1159/000094007, indexed in Pubmed: 16788293.

17. Vlak MH, Algra A, Brandenburg R, et al. Prevalence of unruptured intracranial aneurysms, with emphasis on sex, age, comorbidity, country, and time period: a systematic review and meta-analysis. Lancet Neurol. 2011; 10(7): 626-636, doi: 10.1016/S1474-4422(11)70109-0, indexed in Pubmed: 21641282.

18. Wiebers D. Unruptured intracranial aneurysms: natural history, clinical outcome, and risks of surgical and endovascular treatment. Lancet. 2003; 362(9378): 103-110, doi: 10.1016/s0140-6736(03)13860-3. 